\title{
Prevalence of Nocturia in Sleep-Disordered Breathing and its Correlation with Severity of the Disease
}

\author{
M. Gupta, V. Pilaniya, P. Chatterjee, N. Sood, M. K. Sen, \\ T. Adhikari, J. C. Suri \\ Department of Pulmonary, Critical Care and Sleep Medicine, Vardhmaan Mahavir Medical College and \\ Safdarjung Hospital, New Delhi - 110029, India \\ Doi: 10.5958/j.0973-340X.7.1.006 \\ Indian J Sleep Med 2012; 7.1, 23-28
}

\begin{abstract}
Background: Nocturia is a common complaint in the general population and prevalence varies across studies. It not only causes significant sleep disruption and daytime consequences such as impaired concentration and fatigue but also is independently associated with the risk of cardiovascular morbidity. Nocturia may be associated with various disorders including sleep-disordered breathing (SDB). The mechanism by which SDB causes nocturia is not clear from the various studies conducted so far. Several different hypotheses suggesting various different mechanisms have been proposed. A systematic large-scale study of the association between nocturia and SDB indicating its prevalence and association with its severity has not been performed.

Objective: To investigate the occurrence of nocturia in SDB, identify its prevalence and explore its correlation with severity of the disease.

Methodology: A prospective case-controlled study was conducted among 150 adult patients of SDB with obstructive sleep events, along with 150 age- and sex-matched healthy adult controls. The cases were subclassified into mild (respiratory disturbance index [RDI] $>5-15$ / h), moderate (RDI $>15-30 / h$ ) and severe (RDI $>30 / h$ ) categories comprising 50 patients each. Scoring was done and the diagnosis was made in accordance with the latest American Academy of Sleep Medicine guidelines. Both cases and controls were evaluated for the presence of nocturia, pathological nocturia (PN) and frequency of nocturia through a common questionnaire. Nocturia was defined as self-reported awakening at least once per night to urinate, while PN was defined as awakening at least twice per night to urinate. Statistical analysis was carried out using SPSS 16.0 (SPSS Inc., Chicago, IL USA) and GraphPad Prism 6.01 software and a $p$ value of $<0.05$ was considered as statistically significant.

Results: Nocturia was found to be present in more than $4 / 5$ of the patients, and half of the patient population had PN $(p<0.0001)$. The mean frequency of nocturia in patients was $1.75 \pm 1.29$ and that in the control group was $0.51 \pm .071(p<0.0001)$. Nocturia was found to be more common in females compared with males. The prevalence of PN differed significantly $(p<0.05)$ between various severities of SDB, thereby showing an increase in the
\end{abstract}

Address for Correspondence

Dr J C Suri

Consultant, Professor \& Head

Department of Pulmonary, Critical Care and

Sleep Medicine, Vardhmaan Mahavir Medical

College and Safdariung Hospital, New Delhi 
frequency with increasing severity of the disease. The correlation between severity of SDB and frequency of nocturia was also found to be positive $(p<0.001)$.

Conclusions: Nocturia and PN are more common in patients of SDB as compared to the general population, and more commonly seen with increased severity of SDB. The frequency of nocturia shows an independent positive correlation with severity of SDB irrespective of the presence of various confounders for nocturia. High prevalence of both PN and SDB in the population suggests the likelihood of the presence of a large percentage of patients with nocturia having concomitant SDB.

Keywords: Sleep-disordered breathing, Obstructive sleep apnoea, Hypopnoea syndrome, Respiratory disturbance index, Polysomnography, Pathological nocturia

\section{Introduction}

$\mathrm{N}$ octuria is a highly prevalent and bothersome condition, characterized by the need to wake to void at night. It is a common cause of nocturnal awakenings, may lead to sleep maintenance insomnia and can be associated with impaired mental and somatic health, impaired quality of life and even increased mortality. ${ }^{1-6}$ The International Continence Society has defined nocturia as "the number of voids recorded during a night's sleep: each void is preceded and followed by sleep". ${ }^{7}$ The consequences of nocturia are significantly disturbing, causing sleep disruption, daytime fatigue and an approximately two-fold increase in the number of falls at night. Despite being a troubling condition with severe implications, it often goes unreported as a symptom.

Various epidemiological studies suggest nocturia to be highly common across populations.$^{8-14}$ It is most prevalent in older people, but it also affects a significant proportion of younger individuals. The varying prevalence rates in the general population across different studies could be related to the absence of a uniformly accepted definition of the condition and differences in the nature of the study populations.

Nocturia has been found to occur equally in men and women, as the age-standardized prevalence of nocturia (1 or more voids per night) was approximately $40 \%$ for both genders. ${ }^{10-12}$ Nocturia is more common among women at a younger age but the differences disappear by middle age. In men, the prevalence of nocturia increases more rapidly with age than that in women. ${ }^{8-10}$ Prevalence rates in younger men $(20-50$ years) in various studies were 1 or more voids in 11$35.2 \%$ and 2 or more voids in $2-16.6 \%$. Prevalence rates in younger women were 1 or more voids in 20.4$43.9 \%$ and 2 or more voids in $4.4-18 \% .{ }^{13}$ Studies reveal that subjects with ages between 50 and59, between 60 and 69 and 70 years old or over, respectively, have 1.75 , 3.35 and 6.21 times the prevalence of nocturia than that of the 49 years or younger cohort. In older men (older than 70 years), rates were 1 or more voids in $68.9-93 \%$ and 2 or more voids in $29-59.3 \%$. In older women, rates were 1 or more voids in $74.1-77.1 \%$ and 2 or more voids in $28.3-61.5 \%$. Therefore, in the elderly, nocturia was found to be more frequent among men and up to $60 \%$ of older people void 2 or more times per night. ${ }^{12-14}$

The aetiology of nocturia is inadequately understood. Nocturia is frequently attributed to ageing or childbirth in women ${ }^{9}$ and to benign prostatic hyperplasia (BPH) in men. ${ }^{8}$ Other related conditions include overactive bladder syndrome, nocturnal polyuria, urinary incontinence, awakening for other reasons such as anxiety and use of diuretics, caffeine or alcohol. Nocturia is also found to be related to disorders such as diabetes, cardiovascular disease, chronic renal insufficiency, urinary tract infection and SDB, particularly obstructive sleep apnoea hypopnea syndrome (OSAHS)..$^{3,5,11-13}$

Among the variety of disorders causing nocturia, the association between SDB and nocturia has been least studied. ${ }^{14-18}$ Nocturia is a reported sleep problem in 60\% of cases hospitalized for various sleep-related problems including SDB. Studies report that $76 \%$ of nocturnal urination episodes are immediately preceded by an episode of obstructive apnoea or snoring. ${ }^{19,20}$ The frequency of nocturia has been reported to be greater in adult men with OSAHS than in age-controlled healthy male adults and also has been positively correlated with the severity of OSAHS. ${ }^{21}$ The mechanism by which SDB leads to nocturia has not been definitively identified with multiple hypotheses suggesting different mechanisms. ${ }^{21,22}$ There seemed a need to perform a large-scale systematic 
and comprehensive study indicating the prevalence of nocturia in SDB and its association with severity of SDB.

The objective of our study was to determine the prevalence of nocturia in adult patients of SDB mainly having obstructive sleep apnoea events compared with the general population and to explore its relationship with different grades of severities of SDB between different gender and age groups. Such information may have implications for OSAHS case finding and severity prediction.

\section{Materials and Methods}

A prospective case-controlled study was designed and conducted among patients who had a level I whole night polysomnographic (PSG) study done between June 2011 and June 2012 at the Department of Pulmonary, Critical Care and Sleep Medicine, Vardhmaan Mahavir Medical College, Delhi. The study was performed after approval from the institutional ethical committee and informed consent from the patients.

A total of 150 adult (age $>30$ years) cases of SDB having obstructive sleep events were enrolled in the study including both males and females. The patients were initially evaluated with a detailed sleep and medical history, physical examination, routine and specific laboratory investigations and sleep questionnaires. Their sleepiness was measured according to the Epworth Sleepiness Scale and the Stanford Sleepiness Scale and were then subjected to PSG and the following parameters were measured: two channels each for electroencepholography (EEG), electrooculography (EOG) and electromyography (EMG), airflow recording through nose and mouth by thermistor and nasal pressure cannula, thoracic and abdominal efforts by plethysmography, oxygen saturation through pulse oximetry and tracheal sound recording by using a microphone attached to the neck.

Obstructive apnoea was defined as cessation of airflow at the nose and mouth, as measured by a thermistor/ nasal cannula, while the respiratory effort continues for at least $10 \mathrm{~s}$. Hypopnea was scored when the nasal pressure signal excursions dropped by $\geq 50 \%$ of the baseline for a duration of at least $10 \mathrm{~s}$ and was associated with a drop of saturation of $\geq 3 \%$ from the pre-event baseline or if it was associated with an arousal. Respiratory effort-related arousal (RERA) was scored when there was a sequence of breaths lasting at least 10 $s$, characterized by increasing respiratory effort or flattening of the nasal pressure waveform, leading to an arousal from sleep when the sequence of breaths did not meet the criteria for an apnoea or hypopnoea. SDB was diagnosed when a patient with symptoms suggesting SDB had a respiratory disturbance index (RDI) (apnoeas + hypopnoeas + RERAs) $\geq 5 /$ h.

The cases were subclassified into mild (RDI $\geq 5-15 /$ h), moderate (RDI $\geq 15-30 / h)$ and severe $(R D I \geq 30 / h$ ) categories comprising 50 patients each. Scoring was done and the diagnosis of OSA was made in accordance with the American Academy of Sleep Medicine guidelines. ${ }^{23}$

During the study period, 150 age- and sex-matched healthy controls were also included in the study. Both cases and controls were evaluated for the presence of nocturia, $\mathrm{PN}$ and frequency of nocturia through a common questionnaire. Nocturia was defined as selfreported awakening at least once per night to urinate, while PN was defined as awakening at least twice per night to urinate. The frequency of nocturia was documented $0-5$ as the number of episodes of nocturnal enuresis in all the cases, with a higher number denoting higher frequency. PSG variable included in the study was RDI, whereas the demographic variables defined for the purpose of this study were age, gender and BMI.

Statistical analysis was carried out using SPSS 16.0 (SPSS, Chicago, USA) and GraphPad Prism 6.01 software. Data was analyzed for distribution pattern and homogeneity of variances. Group t-tests were used to assess the significance of differences between patients with SDB and controls with respect to continuous variables (age, BMI, frequency of nocturia). $c^{2}$ test was used to assess the significance of differences between the two groups with respect to categorical variables (gender, age groups, severity of SDB). Multiple-group comparisons of quantitative variables were made using analysis of variance (ANOVA) and intergroup comparisons were carried out using Bonferroni's test to identify the significantly different groups. Correlation analysis was performed using Spearman's test. Data was presented as mean $\pm S D$ wherever appropriate and a $p$ value of $<0.05$ was considered as statistically significant.

\section{Results}

Of a total of 150 patients analyzed, $112(74.7 \%)$ were males and $38(25.3 \%)$ were females, who were further subgrouped according to the severity of SDB into the

Indian Journal of Sleep Medicine (IJSM), Vol. 7, No. 1, 2012 
mild (50), moderate (50) and severe (50) categories. In the control group comprising 150 age- and sex-matched healthy individuals, there were $115(76.7 \%)$ males and $35(23.3 \%)$ females. There was a weak correlation between RDI and age as well as between RDI and BMI $(r=0.2 ; p<0.05)$. The various demographic parameters are shown in Table 1.

Table 1: Demographic Parameters

\begin{tabular}{|l|l|l|l|}
\hline & $\begin{array}{l}\text { Patients } \\
(N=150)\end{array}$ & $\begin{array}{l}\text { Controls } \\
(N=150)\end{array}$ & $p$-value \\
\hline Males $(\mathrm{M})$ & $112(74.7 \%)$ & $115(76.7 \%)$ & $\mathrm{ns}$ \\
\hline Females $(\mathrm{F})$ & $38(25.3 \%)$ & $35(23.3 \%)$ & $\mathrm{ns}$ \\
\hline Age $($ years $)$ & $56.7 \pm 10.6$ & $52.2 \pm 10.1$ & $\mathrm{~ns}$ \\
\hline BMI $\left(\mathrm{kg} / \mathrm{m}^{2}\right)$ & $32.3 \pm 5.7$ & $26.2 \pm 5.0$ & $<0.05$ \\
\hline
\end{tabular}

Of the 150 patients, more than $80 \%$ (124) had nocturia; almost two-third of these patients (80) and half (53.3\%) of the entire patient population also had PN. The prevalence of nocturia in the control group was around $40 \%(60)(p<0.0001)$, while that of PN was less than $10 \%(13)(p<0.0001)$. The mean frequency of nocturia among patients was $1.75 \pm 1.29$, while that of the control group was $0.51 \pm 0.71(p<0.0001)$. In the subgroup analysis, nocturia was found to be more common in females (almost 90\%) compared with males $(80 \%)$; although not amounting to statistical significance, the prevalence rates were similar (around 40\%) in the control group for both the genders $(p<0.0001)$. These results have been compiled in Table 2 .

Table 2: Prevalence of Nocturia

\begin{tabular}{|c|c|c|c|}
\hline & $\begin{array}{l}\text { Patients } \\
(N=150)\end{array}$ & $\begin{array}{l}\text { Controls } \\
(N=150)\end{array}$ & $p$-value \\
\hline $\begin{array}{l}\text { Nocturia } \\
\text { Males } \\
\text { Females }\end{array}$ & $\begin{array}{l}124(82.7 \%) \\
90(80.4 \%) 3 \\
4(89.5 \%)\end{array}$ & $\begin{array}{l}60(40 \%) \\
46(40 \%) \\
14(40 \%)\end{array}$ & $\begin{array}{l}<0.0001 \\
<0.0001 \\
<0.0001\end{array}$ \\
\hline $\begin{array}{l}\text { Pathological } \\
\text { nocturia } \\
\text { Males } \\
\text { Females }\end{array}$ & $\begin{array}{l}80(53.3 \%) \\
56(50.0 \%) \\
24(63.2 \%)\end{array}$ & $\begin{array}{l}13(8.7 \%) \\
10(8.5 \%) \\
3(8.6 \%)\end{array}$ & $\begin{array}{l}<0.0001 \\
<0.0001 \\
<0.0001\end{array}$ \\
\hline $\begin{array}{l}\text { Frequency } \\
\text { of nocturia }\end{array}$ & $1.75 \pm 1.29$ & $0.51 \pm 0.71$ & $<0.0001$ \\
\hline
\end{tabular}

The patient group was further subgrouped into various categories by gender, age and severities. The mean RDI values in males were 9.7 \pm 2.9 (mild), 21.9 \pm 4.6 (moderate) $48.0 \pm 10.8$ (severe) $(p<0.001)$. Similarly, the
Table 3: Subgroup Analysis in SDB Patients

\begin{tabular}{|l|l|l|l|l|}
\hline SDB Patients & $\begin{array}{l}\text { Prevalence } \\
\text { of Nocturia }\end{array}$ & $p$-value & $\begin{array}{l}\text { Prevalence of } \\
\text { Pathological } \\
\text { Nocturia }\end{array}$ & $p$-value \\
\hline Sex & $90(80.4 \%)$ & ns & $56(50.0 \%)$ & ns \\
Males & $34(89.5 \%)$ & & $24(63.2 \%)$ & \\
Females & & & & \\
\hline Age groups & $18(81.8 \%)$ & $\mathrm{ns}$ & $12(54.5 \%)$ & $\mathrm{ns}$ \\
$30-40$ yrs & $39(86.7 \%)$ & & $24(53.3 \%)$ & \\
$40-50$ yrs & $35(74.5 \%)$ & & $22(46.8 \%)$ & \\
$50-60$ yrs & $32(88.9 \%)$ & & $22(61.1 \%)$ & \\
$60-70$ yrs & & & & \\
\hline Severity & $39(78 \%)$ & $\mathrm{ns}$ & $20(40 \%)$ & $\mathrm{p}<0.05$ \\
Mild & $40(80 \%)$ & & $27(54 \%)$ & \\
Moderate & $45(90 \%)$ & & $33(66 \%)$ & \\
Severe & & & & \\
\hline
\end{tabular}

mean RDI values in females were $8.5 \pm 2.5$ (mild), $22.1 \pm 4.8$ (moderate) $40.1 \pm 14.1$ (severe) $(p<0.001)$. The subgroup analysis for the prevalence of nocturia and $\mathrm{PN}$ was performed. The prevalence of PN differed significantly $(p<0.05)$ between various severities of SDB. The prevalence of nocturia and PN was higher with more severe disease, and there was a significant difference in the frequencies of nocturia across different severities, showing an increase in the frequency with increasing severity of the disease. The results of the subgroup analysis have been summarized in Table 3 and Figure 1.

The correlation between the severity of SDB and frequency of nocturia was found to be positive and was statistically significant $(N=150 ; p<0.001)$. Patients with

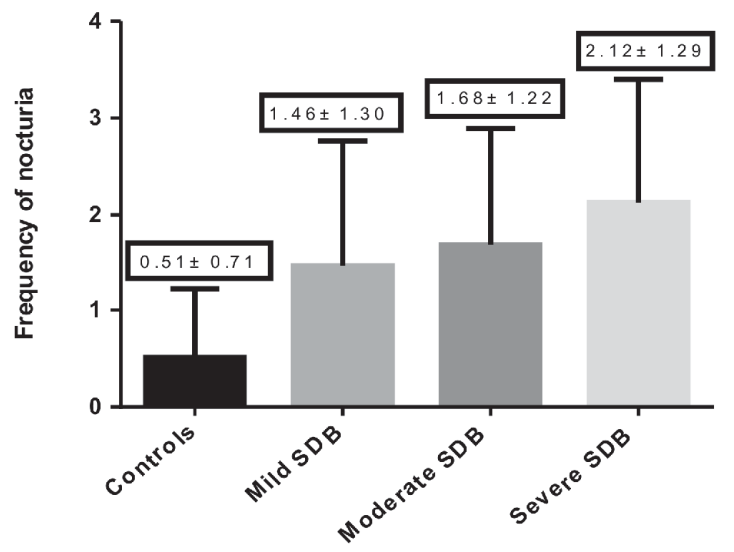

Figure 1: Comparison of frequency of nocturia between various groups control vs mild, moderate, severe $(\mathrm{p}<0.0001)$, mild vs severe $(\mathrm{p}=0.001)$, moderate vs severe $(\mathrm{p}<0.05)$ 
the presence of confounding factors for nocturia (i.e., diabetes, chronic renal disease, congestive heart failure, benign prostatic hyperplasia, cystitis, chronic urinary tract infections and the use of drugs like diuretics) were not considered, and a correlation analysis of the pure group $(N=50)$ between severity of SDB and frequency of nocturia revealed a much stronger positive correlation between the two $\left(r_{\mathrm{s}}=0.7 ; p<0.0001\right)$.

\section{Discussion and Conclusions}

The primary conclusion from our study is that nocturia and $\mathrm{PN}$ are more common in patients with $\mathrm{SDB}$ ( age 30-70 years) compared with the age-matched general population. The prevalence of nocturia and PN, 82.7\% and $53.3 \%$, respectively, in our patients is similar to that reported in elderly (age $>70$ years) populations $(70$ $85 \%$ and $40-60 \%),{ }^{20-22}$ while the prevalence of nocturia and $\mathrm{PN}$ is $40 \%$ and $9 \%$, respectively, in the age-matched general population. Prevalence of nocturia and PN seems to be higher in females than that in males, although not statistically significant because of the fewer number of female patients enrolled in the study; however, the role of age and BMI as predictors of nocturia could not be confirmed through our study.

Nocturia and PN are more commonly seen with the increased severity of SDB as established by our study. The frequency of nocturia shows a positive correlation with the severity of SDB irrespective of the presence of various confounders for nocturia, suggesting that the higher the severity of the disease, the higher the frequency of nocturia in these patients.

Our study was a case-controlled design; therefore, it provides for various advantages over the previously attempted uncontrolled studies. The aim was to maximize the sample size while recruiting adequate controls for comparison. We also decided to collect data of equal number of patients in each subgroup of SDB severity for ease of analysis. However, although large in comparison with the previous published studies, our sample size may not be sufficient to address all of the potentially important subgroup analyses. There also appears to be a male bias in recruitment, which can be attributed to the lesser OPD attendance of female SDB patients in our setting during the specified time period for our study.

Our study was not designed to define the mechanisms for PN in SDB patients, but our data provides some insights with regard to the high prevalence of PN observed in these patients. The mechanism of nocturia in patients with SDB is probably multifactorial. Patients with massive obesity (risk factor for OSAHS) have an increased glomerular filtration rate, higher fractional urinary flows, higher fractional sodium and chloride excretion and a lower percentage of filtered sodium reabsorption compared with normal subjects. ${ }^{24,25}$ Second, SDB leads to disturbed sleep, sleep fragmentation and insufficient arousal response, which itself may be associated with nocturia. ${ }^{26}$ Third, there appears to role for atrial natriuretic peptide (ANP) and antidiuretic hormone $(\mathrm{ADH})$ in these changes in renal function. SDB leads to repetitive episodes of complete or partial upper airway obstruction causing various complex adverse pathophysiological consequences including multiple nocturnal episodes of hypoxemia, increased sympathetic activity (due to hypoxaemia and arousals from sleep), right atrial stretching secondary to increased atrial transmural pressure and exaggerated intrathoracic pressure swings causing increased venous return. Such pathophysiological mechanisms stimulate the secretion of ANP and decreased $\mathrm{ADH}^{27-29}$, thereby cause nocturia. Lastly, the increased intraabdominal pressure caused by respiratory efforts against an obstructed airway can be transmitted to the urinary bladder causing a steep rise in the bladder pressures. Thus, SDB also promotes nocturia by increasing bladder pressure during sleep..$^{30,31}$

There are various other potential aetiologies for nocturia such as diabetes, chronic renal insufficiency, recurrent urinary tract infections, the presence of prostatic enlargement and parity that were not specifically addressed in our study by multivariate analysis. Finally, there is no scientifically validated definition of PN. The definition we used is arbitrary. However, it has been used by others in various studies. ${ }^{20-22,31,32}$

In summary, based on our findings, we conclude that nocturia is highly common in SDB especially OSAHS, affecting almost $80 \%$ of the patients with half of these patients having PN. Considering the high prevalence of $\mathrm{SDB}$ in the general population (2-13.2\%), nocturia is a significant symptom that may affect up to $0.1-7 \%$ of the population. Furthermore, in view of the high prevalence rates of both $\mathrm{PN}$ and SDB, it is likely that a notable percentage of patients with nocturia also have SDB. As shown from our study, nocturia is independently associated with SDB. Some studies have suggested an association between nocturia and cardiovascular morbidity. ${ }^{30-32}$ These results support screening for SDB 
in all patients reporting with nocturia. Increased awareness in this regard will not only improve SDB case finding but also will facilitate treatment strategies for nocturia that are directed to the underlying aetiology in patients who are diagnosed with concomitant SDB.

\section{References}

1. Middelkoop HA, Smilde-van den Doel DA, Neven AK, et al. Subjective sleep characteristics of 1,485 males and females aged 50-93: effects of sex and age, and factors related to self-evaluated quality of sleep. J Gerontol A BiolSci Med Sci 1996; 51:108-115.

2. Asplund R. Mortality in the elderly in relation to nocturnal micturition. BJU Int 1999; 84:297-301.

3. Weiss JP, Blaivas JG. Nocturia. J Urol 2000; 163:5-12.

4. Asplund R, Marnetoft SU, Selander J, et al. Nocturia in relation to somatic health, mental health and pain in adult men and women. BJU Int 2005; 95:816-819.

5. Tikkinen KA, Auvinen A, Johnson TM 2nd, et al. A systematic evaluation of factors associated with nocturia the population based FINNO study. Am J Epidemiol 2009; 170:361-368

6. Tikkinen KA, Johnson TM 2nd, Tammela TL, et al. Nocturia frequency, bother and quality of life: how often is too often? A population-based study in Finland. Eur Urol 2010; 57:488-496.

7. Van Kerrebroeck P, Abrams P, Chaikin D, et al. The standardisation of terminology in nocturia: report from the Standardisation Sub-committee of the International Continence Society. Neurourol Urodyn 2002; 2: 179-183.

8. Malmsten UG, Milsom I, Molander U, Norlen LJ. Urinary incontinence and lower urinary tract symptoms: an epidemiological study of men aged 45 to 99 years. J Urol 1997; 158:1733-1737.

9. Swithinbank LV, Donovan J, James $M C$, Yang $Q$, Abrams P. Female urinary symptoms: age prevalence in a community dwelling population using a validated questionnaire. Neurourol Urodyn 1998; 16:432-434.

10. Yoshimura K, Terada N, Matsui Y, Terai A, Kinukawa N, Arai Y. Prevalence of and risk factors for nocturia: Analysis of a health screening program. Int J Urol 2004; 1 1:282-287.

11. Hunskaar S. Epidemiology of nocturia. BJU Int 2005; 96:4-7.

12. Tikkinen KA, Tammela $T L$, Huhtala $H$, et al. Is nocturia equally common among men and women? A population based study in Finland. J Urol 2006; 175:596-600.

13. Bosch JL, Weiss JP. The prevalence and causes of nocturia. J Urol 2010; 184:440-446.

14. Van Kerrebroeck P. Nocturia: current status and future perspectives. Curr Opin Obstet Gynecol 201 1; 23:376-385.

15. Yokoyama O, Lee SW, Ohkawa M, Amano T, Ishiura Y, Furuta $\mathrm{H}$. Enuresis in an adult female with obstructive sleep apnea. Urology 1995; 45:150-154.
16. Ulfberg $\mathbf{J}$, Thuman R. A non-urologic cause of nocturia and enuresis - obstructive sleep apnea syndrome (OSAS). Scand J Urol Nephr 1996; 30:135-137.

17. Umlauf $\mathbf{M}$, Kurtzer E, Valappil T, Burgio K, Pillion D, Goode P. Sleep-disordered breathing as a mechanism for nocturia: preliminary findings. Ostomy Wound Manage 1999; 45:52-60.

18. Cole OJ, Osborn DE, Hanning CD. Obstructive sleep apnoea causing nocturia. BJU Int 2001; 87:414.

19. Krieger J, Petiau C, Sforza E, Delanoe C, Hecht MT Chamouard V. Nocturnal pollakiuria is a symptom of obstructive sleep apnea. Urologia Internationalis 1993; 50:9397.

20. Pressman MR, Figueroa WG, Kendrick-Mohamed J, Greenspon LW, Peterson DD. Nocturia: A rarely recognized symptom of sleep apnea and other occult sleep disorders. Arch Internal Med 1996; 156:545-550.

21. Hajduk IA, Strollo PJ Jr., Jasani RR, Atwood CW Jr., Houck $P R$, et al. Prevalence and predictors of nocturia in obstructive sleep apnea-hypopnea syndrome - a retrospective study. Sleep 2003; 26:61-64.

22. Umlauf MG, Chasens ER. Sleep disordered breathing and nocturnal polyuria: nocturia and enuresis. Sleep Med Rev 2003; 7:403-411.

23. American Academy of Sleep Medicine. The International Classification of Sleep Disorders: Diagnostic and Coding Manual, 2nd ed, American Academy of Sleep Medicine, Westchester, Illinois 2005.

24. Follenius $\mathbf{M}$, Krieger J, Krauth $\mathbf{M O}$, et al. Obstructive sleep apnea treatment: peripheral and central effects on plasma renin activity and aldosterone. Sleep 1991; 14:211-217.

25. Fletcher EC. Obstructive sleep apnea and the kidney. J Am Soc Nephrol 1993; 4:1111-1121.

26. Berry RB, Kouch KG, Der DE, et al. Sleep apnoea impairs the arousal response to airway occlusion. Chest 1996; 109: 1490-1496.

27. Ichioka $\mathbf{M}$, Hirata $Y$, Inase $N$, Tojo $N$, Yoshizawa $M$, Chida M, Miyazato I, Taniai S, Marumo F. Changes of circulating atrial natriuretic peptide and antidiuretic hormone in obstructive sleep apnea syndrome. Respiration. 1992; 59: 164-168.

28. Lin C, Tsan K. Plasma levels of atrial natriuretic factor in moderate to severe obstructive sleep apnoea syndrome. Sleep 1993; 16: 37-39.

29. Kramer NR, Bonitati AE, Millman RP. Enuresis and obstructive sleep apnea in adults. Chest 1998; 114:634-637.

30. Nogaard JP, Ritting S, Diurhuus JC. Nocturnal enuresis: an approach to treatment based on pathogenesis. J Pediatr 1989; 114:705-710.

31. Romero E, Krakow B, Haynes P, Ulibarri V. Nocturia and snoring: predictive symptoms for obstructive sleep apnea. Sleep Breath 2009; 11:167-172.

32. Parthasarathy S, Fitzgerald MP, Goodwin JL, Unruh $M$, Guerra S, Quan SF. Nocturia, Sleep-disordered breathing, and cardiovascular morbid in a community-based cohort. PLOS ONE 2012; 7: e30969. doi:10.1371/ journal.pone.0030969. 